\title{
Adaptabilidade e estabilidade de genótipos precoces de cana-de-açúcar no Rio Grande do Sul
}

\author{
Mario Alvaro Aloisio Verissimo(1), Sérgio Delmar dos Anjos e Silva(2), \\ Rogério Ferreira Aires ${ }^{(3)}$, Edelclaiton Daros ${ }^{(4)}$ e Wildon Panziera(1)
}

\begin{abstract}
(1)Universidade Federal de Pelotas, Campus Universitário, Caixa Postal 354, CEP 96010-900 Capão do Leão, RS. E-mail: maaverissimo@gmail.com, panziera2@yahoo.com.br (2)Embrapa Clima Temperado, Caixa Postal 403, CEP $96010-971$ Pelotas, RS. E-mail: sergio.anjos@cpact.embrapa.br ${ }^{(3)}$ Fundação Estadual de Pesquisa Agropecuária, Fepagro Nordeste, BR 285, Km 5, Caixa Postal 20, CEP 95200-000 Vacaria, RS. E-mail: rogerio-aires@fepagro.rs.gov.br ${ }^{(4)}$ Universidade Federal do Paraná, Departamento de Fitotecnia e Fitossanitarismo, Rua dos Funcionários, oㅜ 1.540, CEP 80035-050 Curitiba, PR. E-mail: ededaros@ufpr.br
\end{abstract}

Resumo - O objetivo deste trabalho foi avaliar a adaptabilidade e estabilidade de genótipos de cana-de-açúcar de maturação precoce, quanto à produtividade de colmos e de Brix, no Estado do Rio Grande do Sul. Quinze genótipos de maturação precoce foram avaliados nas safras 2009/2010 e 2010/2011, por meio da análise de efeitos principais aditivos e interações multiplicativas (AMMI). As interações genótipo x ambiente foram de alta significância e evidenciaram a alteração de posição na classificação dos genótipos de um ambiente para outro. Novos genótipos superaram, em produtividade e estabilidade, o genótipo-padrão precoce RB855156, embora este ainda se destaque pela produtividade, estabilidade moderada e ampla adaptabilidade. O genótipo RB966928 destacou-se pela riqueza e produtividade de Brix, estabilidade moderada e ampla adaptabilidade. Salto do Jacuí, RS, é o ambiente mais indicado para testes preliminares de seleção de genótipos.

Termos para indexação: Saccharum, AMMI, interação genótipo x ambiente, melhoramento vegetal.

\section{Adaptability and stability of early sugarcane genotypes in Rio Grande do Sul, Brazil}

\begin{abstract}
The objective of this work was to evaluate the adaptability and stability of early maturation sugarcane genotypes, as to yield of stalk and Brix, in Rio Grande do Sul state, Brazil. Fifteen early-maturing genotypes were evaluated in the 2009/2010 and 2010/2011 seasons, through the additive main effects and multiplicative interaction (AMMI) analysis. Genotype $\mathrm{x}$ environment interactions were highly significant and evidenced changes in the genotype ranking according to the environment. New genotypes exceeded the standard early genotype RB855156 for productivity and stability, although it still stands out for its high productivity, moderate stability and wide adaptability. Genotype RB966928 stood out for its richness and yield of Brix, moderate stability and wide adaptability. Salto do Jacuí, RS, Brazil, is the most suitable site for preliminary tests of genotype selection.
\end{abstract}

Index terms: Saccharum, AMMI, genotype x environment interaction, plant breeding.

\section{Introdução}

A área cultivada com cana-de-açúcar no Rio Grande do Sul (RS) ocupa cerca de 37 mil hectares (Instituto Brasileiro de Geografia e Estatística, 2010). Deste total, somente 10 mil hectares são utilizados com fins comerciais à produção de etanol, aguardente, açúcar mascavo, melado e rapadura. O restante é destinado para consumo na propriedade familiar (Emater, 2011). Em 2009, o zoneamento agroecológico da cana-de-açúcar apontou 1,5 milhões de hectares com aptidão ao cultivo no RS (Manzatto et al., 2009).

A diferenciação climática combinada ao tipo de relevo mais abrupto e o destino da produção, bem como o diferencial no processo histórico de colonização, são alguns fatores que vincularam o plantio da cana-de-açúcar à propriedade agrícola familiar gaúcha. A agricultura familiar representa uma grande parcela da economia do Rio Grande do Sul, e a cana-de-açúcar corresponde a uma importante fonte de renda para muitas famílias (Maluf et al., 2008).

A avaliação, identificação e indicação de genótipos promissores de cana-de-açúcar são de fundamental importância para o desenvolvimento da cultura. Uma das ferramentas para auxiliar essa etapa do melhoramento genético da cana-de-açúcar é o estudo da interação genótipo $\mathrm{x}$ ambiente (GxA). Neste estudo, 
busca-se conhecer o comportamento dos genótipos quanto à adaptabilidade - que se refere à capacidade de os genótipos responderem de forma positiva ao estímulo do ambiente -, e à estabilidade, que é a capacidade de os genótipos desempenharem um comportamento previsível em consequência de estímulos ambientais (Cruz \& Carneiro, 2003).

Os estudos a respeito da interação GxA têm sido objeto de diversas pesquisas, em programas de melhoramento genético da cana-de-açúcar, no Brasil (Raizer \& Vencovsky, 1999; Silva et al., 2002; Silva, 2008). A avaliação da interação GxA, por meio da análise de efeitos principais aditivos e interações multiplicativas (AMMI), vem sendo aplicada com sucesso a diversas culturas (Pereira et al., 1998; Oliveira et al., 2003; Melo et al., 2007; Arciniegas-Alarcón \& Dias, 2009; Marjanović-Jeromela et al., 2011), assim como na cana-de-açúcar (Guerra et al., 2009; Silveira, 2011). Este modelo combina um método univariado para os efeitos aditivos de genótipos e ambientes, com um método multivariado para o efeito multiplicativo da interação GxA (Zobel et al., 1988). Por conseguinte, pode contribuir tanto para a identificação de genótipos amplamente adaptados, com alto rendimento, como para o zoneamento agronômico, a recomendação de cultivares regionais e a escolha de locais de teste (Gauch \& Zobel, 1996).

O objetivo deste trabalho foi avaliar a adaptabilidade e estabilidade de genótipos de cana-de-açúcar de maturação precoce, quanto à produtividade de colmos e de Brix, no Rio Grande do Sul, por meio da análise AMMI.

\section{Material e Métodos}

A avaliação da adaptabilidade e estabilidade de 15 genótipos de maturação precoce foi realizada em nove locais, no Rio Grande do Sul, em cultivo de cana-planta, soca 1 e soca 2, no total de 18 ambientes (Tabela 1). Foram analisados os dados de produtividade das safras 2009/2010 e 2010/2011, provenientes de experimentos de avaliação de genótipos de cana-de-açúcar, introduzidos pela Embrapa Clima Temperado, desenvolvidos pela Rede Interuniversitária para o Desenvolvimento do Setor Sucroenergético (Ridesa) e disponibilizados pelo Programa de Melhoramento Genético da Cana-de-açúcar (PMGCA) da Universidade Federal do Paraná.
Para o plantio, foi adotado o sistema manual (convencional), pelo qual os toletes com três gemas foram distribuídos dentro dos sulcos, tendo sido colocados seis toletes por metro linear, no total de 18 gemas por metro. O plantio foi realizado entre agosto e outubro, e a colheita após 11 a 13 meses. No pré-plantio, o solo foi preparado com uma aração e duas gradagens; em seguida, procedeu-se à formação dos sulcos, com 30 a $40 \mathrm{~cm}$ de profundidade e $1,40 \mathrm{~m}$ entre sulcos. A adubação de plantio foi feita com $60 \mathrm{~kg} \mathrm{ha}^{-1}$ de N, $120 \mathrm{~kg} \mathrm{ha}^{-1}$ de $\mathrm{P}_{2} \mathrm{O}_{5}$ e $120 \mathrm{~kg} \mathrm{ha}^{-1}$ de $\mathrm{K}_{2} \mathrm{O}$. Entre dezembro e janeiro, foi realizada a adubação de cobertura, com $100 \mathrm{~kg} \mathrm{ha}^{-1}$ de $\mathrm{N}$. No cultivo de cana-soca, foi realizada uma adubação na entrelinha com $\mathrm{N}$ e $\mathrm{K}$, após a colheita dos experimentos, nas doses de $100 \mathrm{~kg} \mathrm{ha}^{-1}$ de $\mathrm{N}$ e $120 \mathrm{~kg} \mathrm{ha}^{-1}$ de $\mathrm{K}_{2} \mathrm{O}$.

Utilizou-se o delineamento experimental de blocos ao acaso, com três repetições. As parcelas foram constituídas de duas a seis linhas de oito metros de comprimento. As variáveis utilizadas para a análise AMMI foram produtividade de colmos $(\mathrm{TCH}$, $\mathrm{Mg} \mathrm{ha}^{-1}$ ) e de Brix ( $\mathrm{TBH}, \mathrm{Mg} \mathrm{ha}^{-1}$ ). O cálculo da $\mathrm{TCH}$ foi realizado por meio da equação $\mathrm{TCH}=(\mathrm{P} 10 \mathrm{C} / 10) \mathrm{x}$ NCM x (10/E) em que: P10C é a massa de 10 colmos (kg); NCM é o número de colmos por metro; e E é o espaçamento $(1,4 \mathrm{~m})$. Procedeu-se às leituras de Brix (percentagem de massa/massa dos sólidos solúveis contidos em uma solução) em três diferentes colmos, em amostragem realizada em dois pontos por colmo, no terceiro entrenó abaixo do ponto de quebra e terceiro acima do solo, por meio de refratômetro manual. Para o cálculo do $\mathrm{TBH}$, utilizou-se a equação $\mathrm{TBH}=$ (valor médio do Brix x TCH)/100.

As análises estatísticas foram realizadas em separado para TCH e TBH. Primeiramente, procedeu-se à análise de variância de cada ambiente (análises individuais). Uma vez detectada a diferença entre os tratamentos, realizou-se a análise de variância conjunta (Ramalho et al., 2000), implementada mesmo não tendo havido homogeneidade entre as variâncias residuais $\left(\mathrm{QM}_{\mathrm{R}}\right)$. Assim, procedeu-se ao ajuste dos graus de liberdade do erro médio e da interação GxA, segundo o método de Cochran (1954). Após os ajustes, procedeu-se às interpretações relativas às significâncias do teste F.

Realizada a análise de variância conjunta, e detectada a significância para a fonte de variação da interação GxA, procedeu-se à análise de adaptabilidade e estabilidade, por meio da AMMI (Zobel et al., 1988), 
conforme o seguinte modelo, descrito por Duarte \& Vencovsky (1999): $\mathrm{Y}_{\mathrm{ij}}=\mu+\mathrm{g}_{\mathrm{i}}+\mathrm{a}_{\mathrm{j}}+\sum_{\mathrm{k}=1} \lambda_{\mathrm{k}} \gamma_{\mathrm{ik}} \alpha_{\mathrm{jk}}$ $+\rho_{\mathrm{ij}}+\overline{\mathrm{e}}_{\mathrm{ij}}$, em que: $\mathrm{Y}_{\mathrm{ij}}$ é a resposta média do genótipo $\mathrm{i}$ $(\mathrm{i}=1,2, \ldots, \mathrm{G}$ genótipos), no ambiente $\mathrm{j}(\mathrm{j}=1,2, \ldots$, A ambientes); $\mu$ é a média geral dos ensaios; $g_{i}$ é o efeito fixo do genótipo $i ; a_{j}$ é o efeito fixo do ambiente j. A interação GxA é moderada pelos fatores: $\lambda_{k}$, que é o valor singular do k-ésimo componente principal da interação (IPCA; $\mathrm{k}=1,2, \ldots \mathrm{p}$, em que $\mathrm{p}$ é o número máximo de componentes principais estimáveis); $\gamma_{\mathrm{ik}}$ é o valor singular do j-ésimo ambiente, no k-ésimo IPCA; $\alpha_{\mathrm{ik}}$ é o valor singular do i-ésimo genótipo, no k-ésimo IPCA; $\mathrm{k}$ são as raízes características não nulas, com a expressão $\mathrm{k}=[1,2, \ldots \min (\mathrm{g}-1$, e- 1$)]$; $\rho_{\mathrm{ij}}$ é o resíduo da interação GxA ou resíduo AMMI (ruído presente nos dados); $\overline{\mathrm{e}}_{\mathrm{ij}}$ é o erro experimental médio assumido independentemente.

A análise AMMI pode gerar uma família de modelos (AMMI0, AMMI1, AMMI2, ... , AMMIn), ou seja, combinação das médias e eixos principais, que captam porções da variação da matriz GA, conforme descrito anteriormente. Os procedimentos, métodos e critério de escolha do modelo AMMI a ser adotado estão descritos em Duarte \& Vencovsky (1999), inclusive uma rotina computacional em linguagem SAS/IML para realização das análises. As coordenadas de genótipos e de ambientes, nos eixos principais (IPCA), são representadas em um gráfico biplot (Gabriel, 1971). As análises de variância, a análise AMMI e os gráficos biplot foram realizados com o auxílio do programa SAS 9.2 (SAS Institute, 2009).

Um referencial teórico, complementar aos genótipos e ambientes avaliados pela análise AMMI, foi inserido e denominado genótipo e ambiente suplementar ou ideal (Gi e Ai), com base na proposição de Lin \& Binns (1988), conforme descrito em Pacheco et al. (2005). Segundo essa abordagem, a superioridade agronômica de um genótipo deve ser avaliada relativamente às produtividades máximas observadas em cada ambiente. Assim, um genótipo teoricamente ideal seria aquele que reunisse, ao longo dos ambientes de teste, essas produtividades máximas.

\section{Resultados e Discussão}

A análise de variância mostrou que todos os efeitos avaliados (genótipo, ambiente e interação GxA) foram significativos (Tabela 2) para as duas variáveis analisadas. Esse resultado indica que os genótipos apresentaram comportamento diferenciado conforme as mudanças proporcionadas pelos ambientes. Portanto, o estudo mais detalhado dessa interação se faz necessário,

Tabela 1. Localização, identificação de ambiente, identificação de genótipo, empresa parceira, safra e ciclo de cultivo dos 18 ambientes no Rio Grande do Sul, RS, onde os 15 genótipos de maturação precoce de cana-de-açúcar foram avaliados em 2009-2011.

\begin{tabular}{|c|c|c|c|c|c|c|c|}
\hline Local & ID & Coordenadas geográficas & Empresa & Safra & Ciclo & Genótipo & ID \\
\hline Caxias do Sul & A1 & $29^{\circ} 07^{\prime} 57^{\prime \prime S} ; 52^{\circ} 00^{\prime} 21^{\prime \prime W}$ & Fepagro & $2010 / 2011$ & Planta & $\mathrm{RB} 855156^{(1)}$ & G1 \\
\hline Erechim & A2 & $27^{\circ} 36^{\prime} 40^{\prime \prime} \mathrm{S} ; 52^{\circ} 13^{\prime} 37^{\prime \prime} \mathrm{W}$ & URI & $2010 / 2011$ & Planta & RB925211 & G2 \\
\hline Pelotas & A3 & $31^{\circ} 41^{\prime} 08^{\prime \prime} \mathrm{S} ; 52^{\circ} 25^{\prime} 56^{\prime \prime} \mathrm{W}$ & Embrapa & $2009 / 2010$ & Soca1 & RB925345 & G3 \\
\hline Pelotas & A4 & $31^{\circ} 41^{\prime} 06^{\prime \prime S} ; 52^{\circ} 26^{\prime} 06^{\prime \prime} \mathrm{W}$ & Embrapa & $2010 / 2011$ & Planta & RB935581 & G4 \\
\hline Pelotas & A5 & $31^{\circ} 41^{\prime} 08^{\prime \prime S} ; 52^{\circ} 25^{\prime} 56^{\prime \prime} \mathrm{W}$ & Embrapa & $2010 / 2011$ & Soca 2 & RB946903 & G5 \\
\hline Porto Xavier & A6 & $27^{\circ} 53^{\prime} 34^{\prime \prime} \mathrm{S} ; 55^{\circ} 10^{\prime} 59^{\prime \prime} \mathrm{W}$ & Coopercana & $2009 / 2010$ & Soca 1 & RB965902 & G6 \\
\hline Porto Xavier & A7 & $27^{\circ} 53^{\prime} 06^{\prime \prime S} ; 55^{\circ} 10^{\prime} 58^{\prime \prime} \mathrm{W}$ & Coopercana & $2010 / 2011$ & Planta & RB965911 & G7 \\
\hline Porto Xavier & A8 & $27^{\circ} 53^{\prime} 34^{\prime \prime} \mathrm{S} ; 55^{\circ} 10^{\prime} 59^{\prime \prime} \mathrm{W}$ & Coopercana & $2010 / 2011$ & Soca 2 & RB966923 & G8 \\
\hline São Borja & A9 & $28^{\circ} 41^{\prime} 47^{\prime \prime S} ; 55^{\circ} 57^{\prime} 44^{\prime \prime} \mathrm{W}$ & Fepagro & $2009 / 2010$ & Soca 1 & RB966928 & G9 \\
\hline São Borja & A10 & $28^{\circ} 41^{\prime} 43^{\prime \prime S} ; 55^{\circ} 57^{\prime} 50^{\prime \prime} \mathrm{W}$ & Fepagro & $2010 / 2011$ & Planta & RB975932 & G10 \\
\hline São Borja & A11 & $28^{\circ} 41^{\prime} 47^{\prime \prime S} ; 55^{\circ} 57^{\prime} 44^{\prime \prime} \mathrm{W}$ & Fepagro & $2010 / 2011$ & Soca 2 & RB975935 & G11 \\
\hline Salto do Jacuí & A12 & $28^{\circ} 59^{\prime} 32^{\prime \prime} \mathrm{S} ; 53^{\circ} 13^{\prime} 52^{\prime \prime} \mathrm{W}$ & Grandespe & $2009 / 2010$ & Soca 1 & RB975944 & G12 \\
\hline Salto do Jacuí & A13 & $28^{\circ} 59^{\prime} 25^{\prime \prime S} ; 53^{\circ} 14^{\prime} 23^{\prime \prime} \mathrm{W}$ & Grandespe & $2010 / 2011$ & Planta & RB986419 & G13 \\
\hline Salto do Jacuí & A14 & $28^{\circ} 59^{\prime} 32^{\prime \prime} \mathrm{S} ; 53^{\circ} 13^{\prime} 52^{\prime \prime} \mathrm{W}$ & Grandespe & $2010 / 2011$ & Soca 2 & RB986955 & G14 \\
\hline São Luiz Gonzaga & A15 & $28^{\circ} 22^{\prime} 09^{\prime \prime S} ; 54^{\circ} 54^{\prime} 57^{\prime \prime} \mathrm{W}$ & Norobios & $2009 / 2010$ & Soca 1 & RB996961 & G15 \\
\hline São Luiz Gonzaga & A16 & $28^{\circ} 22^{\prime} 12^{\prime \prime} \mathrm{S} ; 54^{\circ} 55^{\prime} 00^{\prime \prime} \mathrm{W}$ & Norobios & $2010 / 2011$ & Planta & & \\
\hline São Luiz Gonzaga & A17 & $28^{\circ} 22^{\prime} 09^{\prime \prime} \mathrm{S} ; 54^{\circ} 54^{\prime} 57^{\prime \prime} \mathrm{W}$ & Norobios & $2010 / 2011$ & Soca 2 & & \\
\hline$\underline{\text { Santa Rosa }}$ & A18 & $27^{\circ} 50^{\prime} 31^{\prime \prime S} ; 54^{\circ} 26^{\prime} 50^{\prime \prime} \mathrm{W}$ & Coopermil & $2010 / 2011$ & Planta & & \\
\hline
\end{tabular}

(1) Testemunha. 
de modo que ela possa ser controlada ou interpretada e não interfira negativamente na recomendação dos melhores genótipos.

A soma dos quadrados da interação $\left(\mathrm{SQ}_{\mathrm{GxA}}\right)$ foi decomposta em doze eixos de CP; no entanto, somente os cinco primeiros mostraram diferenças significativas ( $<<0,01)$, para ambas as variáveis. Os modelos AMM1 (IPCA1 x TBH ou TCH) e AMMI2 (IPCA1 x IPCA2) foram preferidos para a interpretação e visualização bidimensional (Tabela 2).

As variâncias acumuladas nos dois primeiros IPCA explicaram 42,7 e 40,5\%, para as variáveis TBH e TCH, respectivamente, tendo-se usado, no entanto, menos de $30 \%$ dos graus de liberdade originais. Estes valores estão ligeiramente abaixo dos encontrados para cana-de-açúcar (Bajpai \& Kumar, 2005; Guerra et al., 2009; Silveira, 2011) e semelhantes aos observados por Crossa et al. (1991) em trigo, por Pereira \& Costa (1998) em batata e por Oliveira et al. (2003) em soja. Estes dois modelos explicaram a maior proporção da variação útil (padrão ou efeito de genótipos e ambientes). Portanto, infere-se que os $57 \%$ restantes da $\mathrm{SQ}_{\mathrm{GxA}}$ referentes aos demais eixos, provavelmente, continham pouca informação relevante, isto é, grande parcela da variação considerada "ruído", em respostas imprevisíveis e não interpretáveis.

Para a análise da adaptabilidade e estabilidade dos genótipos de cana-de-açúcar, optou-se por utilizar todos os 18 ambientes avaliados. Porém, alguns ambientes contribuíram fortemente para a interação GxA (Caxias do Sul, Erechim, São Borja e A17 de São Luiz Gonzaga). Tais ambientes apresentaram condições desfavoráveis, seja por falha na brotação em consequência da estiagem, seja por problemas na condução da soqueira (São Borja e A17 de São Luiz Gonzaga), ou pelo efeito do frio (Caxias do Sul e Erechim), que afetaram negativamente as $\mathrm{TCH}$ e TBH. Estes ambientes podem ter influenciado a alta significância da interação GxA e a baixa contribuição dos dois primeiros eixos para a explicação da interação GxA. Mesmo assim, optou-se pela continuidade destes ambientes para interpretação das análises.

As Figuras 1 e 2 mostram os biplots AMMI1 e AMMI2, para TBH e TCH, respectivamente. No modelo AMMI1, a abscissa representa os efeitos principais (médias de genótipos), e a ordenada representa o primeiro eixo de interação (IPCA1). Assim, genótipos com valores de IPCA1 próximos de zero mostraram alta estabilidade aos ambientes de teste. No modelo AMMI2, os genótipos próximos da origem dos eixos são mais estáveis do que aqueles mais afastados, pois contribuíram pouco para a interação; combinações de genótipos e ambientes com escores IPCA de mesmo sinal têm interações específicas positivas; combinações de sinais opostos apresentam interações específicas negativas (Chaves, 2001).

No biplotAMMI1 para TBH (Figura 1), os genótipos de maturação precoce que se posicionaram mais próximos da origem, em relação ao eixo IPCA1, foram RB935581 (G4), RB975932 (G10), RB975944 (G12) e RB986419 (G13), o que indica a alta estabilidade fenotípica para TBH. Entretanto, apenas G4 e G12 obtiveram médias de TBH semelhantes entre si e superiores à média geral, tendo alcançado a sexta e a quinta posição geral,

Tabela 2. Análise de variância conjunta para produtividade de colmo $\left(\mathrm{TCH}, \mathrm{Mg}^{-1}\right)$ e de $\mathrm{Brix}\left(\mathrm{TBH}, \mathrm{Mg}^{-1}\right)$ de 15 genótipos de cana-de-açúcar de maturação precoce, avaliados em 18 ambientes no Rio Grande do Sul, com desdobramento da interação original GxA pelo modelo AMMI, em nível de médias ${ }^{(1)}$, em 2009-2011.

\begin{tabular}{|c|c|c|c|c|c|c|c|c|}
\hline \multirow[t]{2}{*}{ Fonte de variação } & \multicolumn{4}{|c|}{$\mathrm{TCH}$} & \multicolumn{4}{|c|}{$\mathrm{TBH}$} \\
\hline & $\mathrm{GL}^{(2)}$ & QM & $\mathrm{F}$ & $\operatorname{Pr}>\mathrm{F}$ & $\mathrm{GL}^{(2)}$ & QM & $\mathrm{F}$ & $\operatorname{Pr}>\mathrm{F}$ \\
\hline Blocos/ambiente & 30 & 493,7 & - & - & 30 & 19,8 & - & - \\
\hline Genótipos (G) & 14 & $1.566,7$ & 3,93 & 0,000 & 14 & 76,9 & 4,37 & 0,000 \\
\hline Ambiente (A) & 17 & $37.069,8$ & 92,97 & 0,000 & 17 & $1.348,2$ & 76,60 & 0,000 \\
\hline $\mathrm{G} \times \mathrm{A}$ & 238 & 860,7 & 2,16 & 0,001 & 238 & 34,7 & 1,97 & 0,001 \\
\hline Resíduo & 415 & 398,7 & - & - & 415 & 17,6 & - & - \\
\hline Interação GxA (médias) & 238 & 363,19 & 2,16 & 0,001 & 238 & 14,64 & 1,97 & 0,001 \\
\hline IPCA1 (padrão) & 30 & 606,67 & 4,43 & 0,000 & 30 & 33,06 & 4,30 & 0,000 \\
\hline Resíduo AMMI1 & 208 & 285,02 & 2,08 & 0,000 & 208 & 13,76 & 1,79 & 0,000 \\
\hline IPCA2 & 28 & 470,82 & 3,44 & 0,000 & 28 & 23,41 & 3,05 & 0,000 \\
\hline Resíduo AMMI2 & 180 & 256,12 & 1,87 & 0,000 & 180 & 12,26 & 1,60 & 0,000 \\
\hline Erro médio/r & 415 & 168,20 & - & - & 415 & 7,43 & - & - \\
\hline
\end{tabular}

${ }^{(1)} \mathrm{O}$ número de repetições foi 2,37 (média harmônica, em razão do desbalanceamento). (2)Valores ajustados pelo método Cochran (1954), em razão da heterogeneidade de QM residuais. 
respectivamente. A alta estabilidade foi confirmada pelo biplot AMMI2 somente para o genótipo G4, indicada pela proximidade com a origem dos eixos IPCA1 e IPCA2. Este genótipo merece destaque pela alta produtividade de biomassa, pois alcançou a terceira posição geral. Entretanto, carece em riqueza (menor
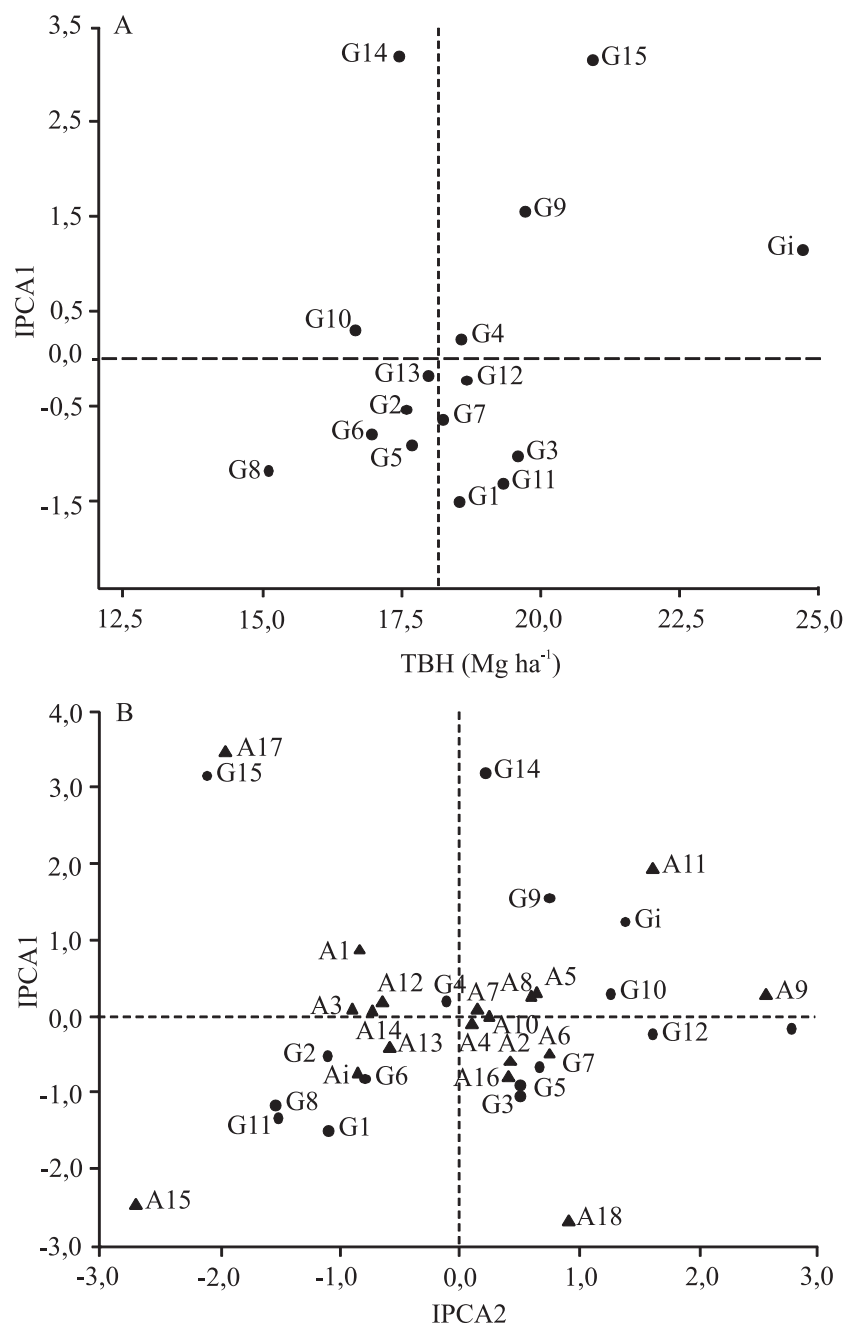

Figura 1. Biplot AMMI1, A, e AMMI2, B, para produtividade de Brix (TBH, $\mathrm{Mg} \mathrm{ha}^{-1}$ ) de 15 genótipos (G) de canade-açúcar de maturação precoce, avaliados em 18 ambientes (A), no Rio Grande do Sul. Genótipos: RB855156 (G1, testemunha), RB925211 (G2), RB925345 (G3), RB935581 (G4), RB946903 (G5), RB965902 (G6), RB965911 (G7), RB966923 (G8), RB966928 (G9), RB975932 (G10), RB975935 (G11), RB975944 (G12), RB986419 (G13), RB986955 (G14), RB996961 (G15) e genótipo ideal (Gi). Ambientes: Caxias do Sul (A1), Erechim (A2), Pelotas (A3, A4 e A5), Porto Xavier (A6, A7 e A8), São Borja (A9, A10 e A11), Salto do Jacuí (A12, A13 e A14), São Luiz Gonzaga (A15, A16 e A17), Santa Rosa (A18) e ambiente ideal (Ai).
${ }^{\circ}$ Brix ou sacarose) no início da safra, fato observado pela sexta posição em TBH.

Os genótipos RB966928 (G9) e RB925345 (G3) diferiram em interação (escores de IPCA1), mas não em média de TBH, enquanto os clones RB986955 (G14) e RB996961 (G15) assemelharam-se em interação
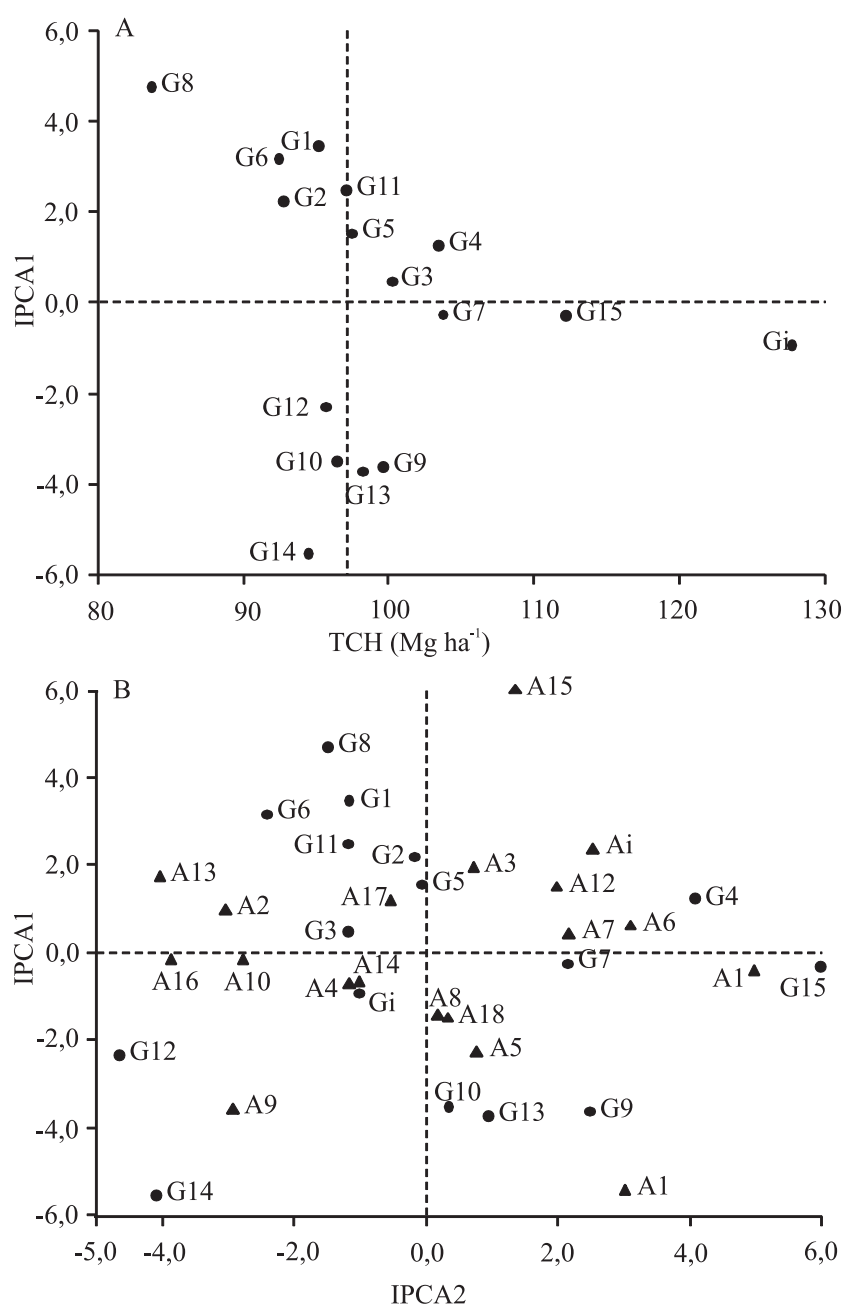

Figura 2. Biplot AMMI1, A e AMMI2, B, para produtividade de colmos ( $\mathrm{TCH}, \mathrm{Mg} \mathrm{ha}^{-1}$ ) de 15 genótipos (G) cana-de-açúcar de maturação precoce, avaliados em 18 ambientes (A), Rio Grande do Sul. Genótipos: RB855156 (G1, testemunha); RB925211 (G2), RB925345 (G3), RB935581 (G4), RB946903 (G5), RB965902 (G6), RB965911 (G7), RB966923 (G8), RB966928 (G9), RB975932 (G10), RB975935 (G11), RB975944 (G12), RB986419 (G13), RB986955 (G14), RB996961 (G15) e genótipo ideal (Gi). Ambientes: Caxias do Sul (A1), Erechim (A2), Pelotas (A3, A4 e A5), Porto Xavier (A6, A7 e A8), São Borja (A9, A10 e A11), Salto do Jacuí (A12, A13 e A14), São Luiz Gonzaga (A15, A16 e A17), Santa Rosa (A18) e ambiente ideal (Ai). 
(baixa estabilidade) e diferiram em produtividade. Os genótipos que mais contribuíram para a interação GxA foram G15, G14, G9, G1, G11 e G3. Os destaques são para RB966928 (G9) e RB925345 (G3) que, em razão de sua riqueza em açúcar, alcançaram alto TBH. O G9 poderia ser identificado como estável, pois foi o genótipo que chegou mais próximo do genótipo ideal (Gi) proposto por Lin \& Binns (1988) e descrito por Pacheco et al. (2005). Resultados semelhantes foram observados por Guerra et al. (2009) no Estado do Paraná, que verificaram médias elevadas de produtividade de sacarose (pol), alta estabilidade fenotípica e adaptabilidade geral para RB966928. Outro comportamento interessante do G9 foram os valores do IPCA1 e 2, semelhantes em número, mas de sinal oposto ao do G1, seu genitor materno (Daros et al., 2010). O G9 mostrou-se, em média, 6,4 e 4,6\% superior ao $\mathrm{G} 1$, quanto a $\mathrm{TBH}$ e $\mathrm{TCH}$, respectivamente, embora G1 tenha superado G9 em seis ambientes (A2, A3, A9, A10, A13 e A15). Neste último ambiente (cultivo de soca 1, em São Luiz Gonzaga, A15), o G1 apresentou a maior média de TBH e TCH, o que indica que este genótipo, considerado como testemunha, mantém produtividade razoável para algumas condições do Rio Grande do Sul.

O clone RB996961 (G15) apresentou a maior média geral de TCH e TBH, porém baixa estabilidade em TBH (Figura 1). A instabilidade deste genótipo é confirmada pelo biplot AMMI2 e deve-se à interação positiva (adaptabilidade específica) com os ambientes Caxias do Sul (A1), Pelotas (A3 e A4), Porto Xavier (A6 e A7), São Borja (A10 e A11), Salto do Jacuí (A12 e A14) e Santa Rosa (A18), e interação negativa com os demais ambientes (Erechim e São Luiz Gonzaga).

No entanto, para TCH, a análise do biplot AMMI1 (Figura 2) mostrou que o G15 possui excelente comportamento, como elevada estabilidade (IPCA1 0 ) e alta produtividade (posição 1), e foi o genótipo que mais se aproximou do ideal (Gi). Sua estabilidade, todavia, não foi confirmada pelo biplot AMMI2. Este comportamento foi ocasionado pela interação negativa com os ambientes A2, A13 e A16, nos locais Erechim, Salto do Jacuí e São Luiz Gonzaga, respectivamente. Segundo Duarte \& Vencovsky (1999), essa discordância é comum nos casos em que resta uma razoável variabilidade, no sentido dos eixos desconsiderados na representação, o que confirma, portanto, que o segundo eixo contém informação relevante para a interação
GxA, uma vez que o problema não teria surgido, se a variabilidade nessa dimensão fosse pequena. Observações semelhantes foram relatadas em estudos de cana-de-açúcar, por Guerra et al. (2009) e Silveira (2011).

Quanto a TCH, o comportamento dos genótipos se alterou ligeiramente (Figura 2). O genótipo RB965911 (G7) alcançou a segunda posição, em razão de seu maior $\mathrm{TCH}$, porém menor $\mathrm{TBH}$ (próximo a média), com adaptabilidade específica aos ambientes de Pelotas, Salto do Jacuí (A12), São Luiz Gonzaga e Santa Rosa. Guerra et al. (2009) observaram comportamento semelhante para este genótipo, com adaptação específica no cultivo de soqueira em alguns locais do Paraná.

O genótipo RB925345 (G3) foi o mais próximo do referencial teórico (Gi) proposto por Lin \& Binns (1988) (Figura 2, AMMI2), comportamento este que era anteriormente ocupado pelo genótipo RB966928 (G9), quanto a TBH. Esta mudança de padrão entre os dois genótipos é atribuída, basicamente, à maior riqueza de G9 e ao elevado potencial de produção de biomassa do G3. No entanto, G3 mostrou-se suscetível ao carvão (Sporisorium scitamineum Syd. sinonímia Ustilago scitaminea Syd.), na ocasião, observado somente em Porto Xavier. É nesse ambiente que se encontra a única usina de etanol do Estado, com cultivo comercial de mais de 20 anos. A pressão de inóculo do carvão, provavelmente, é maior em Porto Xavier do que nos demais ambientes.

A análise AMMI proporcionou a visualização de ambientes similares e mais estáveis, principalmente por meio dos biplots AMMI2. Ambientes similares são aqueles que apresentam escores de IPCA semelhantes. Ambientes estáveis são aqueles onde o ordenamento dos genótipos apresenta maior consistência e confiabilidade, em relação à média dos ambientes. Nesses ambientes, genótipos com adaptabilidade geral tendem a se destacar e podem ser selecionados com maior segurança. Além disso, podem servir de referência para recomendações em outras áreas e, ainda, podem ser os mais adequados para o desenvolvimento de fases preliminares de seleção de cana-de-açúcar (Duarte \& Vencovsky, 1999; Guerra et al., 2009). Portanto, são ambientes mais representativos das condições edafoclimáticas médias para o cultivo da cana-de-açúcar no Rio Grande do Sul. 
Assim, o número de locais de teste pode ser reduzido ou um ambiente pode substituir outro, caso haja necessidade em alguma safra. Porém, é importante dar continuidade aos estudos em ambientes mais estáveis. Desta forma, pode-se obter a melhoria do processo de avaliação de genótipos de cana-de-açúcar, bem como a otimização dos recursos financeiros.

Os ambientes que menos contribuíram para a interação GxA foram Porto Xavier (A6, A7 e A8), Pelotas (A3, A4 e A5) e Salto do Jacuí (A12, A13 e A14) (Figura 1, AMMI2). Portanto, esses ambientes se mostraram mais estáveis e particularmente úteis na avaliação de genótipos para TBH.

Além disso, a indicação de genótipos de cana-de-açúcar para São Luiz Gonzaga, São Borja e Erechim será mais bem sucedida, caso seja específica para esses ambientes. Estes locais também apresentaram o maior distanciamento (interação) entre as safras, o que dificulta ainda mais a indicação de genótipos.

Em São Luiz Gonzaga, os genótipos com adaptação específica foram G3, G5, G6, G9 e G11. O genótipo G12 apresentou alta adaptação somente para o cultivo de cana-planta (1ำ lugar para TBH), por isso, mais estudos devem ser realizados, para conhecer o real comportamento deste genótipo antes de sua indicação para São Luiz Gonzaga.

Para São Borja, a adaptabilidade específica foi verificada pelos genótipos G3, G7, G12, G13, e G14. O genótipo G9 adaptou-se bem somente no ambiente A11 (cultivo de soca 2). E para Erechim, os melhores genótipos foram G3, G7, G11, G14 e G15.

Quanto a TBH, os ambientes Erechim (A2) e São Luis Gonzaga (A16), em cultivo de cana planta, foram aparentemente mais similares. Os valores de $\mathrm{TBH}$ para os ambientes Pelotas (A4) e Porto Xavier (A7) ficaram bem próximos dos de São Borja (A10). Assim, estes ambientes podem ser substituídos numa eventual situação de necessidade.

\section{Conclusões}

1. Os genótipos de cana-de-açúcar de maturação precoce apresentam interação com os ambientes de cultivo.

2. Novos genótipos superam em produtividade e estabilidade o genótipo-padrão precoce RB855156, embora este mostre adaptação específica e estabilidade moderada.
3. O genótipo RB966928 destaca-se pela riqueza e produtividade de Brix, estabilidade moderada e ampla adaptabilidade.

4. Os genótipos RB996961, RB975935, RB975944 e RB935581 apresentam alta produtividade, estabilidade moderada e adaptabilidade específica, com destaque para RB996961, que apresenta a maior produtividade de Brix.

5. Salto do Jacuí é o ambiente mais indicado para testes preliminares de seleção de genótipos de cana-de açúcar, pois apresenta alta estabilidade, o que possibilita o ordenamento de genótipos com maior confiabilidade em relação à média geral dos ambientes testados.

\section{Agradecimentos}

AoConselhoNacionaldeDesenvolvimentoCientífico e Tecnológico, à Coordenação de Aperfeiçoamento de Pessoal de Nível Superior e à Financiadora de Estudos e Projetos, pelo apoio financeiro; à Granja e Destilaria São Pedro, Cooperativa Mista São Luiz Ltda., Noroeste Bioenergética, Cooperativa dos Produtores de Cana Porto Xavier Ltda., Embrapa Clima Temperado, Fundação Estadual de Pesquisa Agropecuária, Universidade Regional Integrada e à Empresa de Assistência Técnica e Extensão Rural, pelo apoio na condução dos experimentos.

\section{Referências}

ARCINIEGAS-ALARCÓN, S.; DIAS, C.T. dos S. Análise AMMI com dados imputados em experimentos de interação genótipo $\mathrm{x}$ ambiente de algodão. Pesquisa Agropecuária Brasileira, v.44, p.1391-1397, 2009.

BAJPAI, P.K.; KUMAR, R. Comparison of methods for studying genotype x environment interaction in sugarcane. Sugar Tech, v.7, p.129-135, 2005.

CHAVES, L.J. Interação de genótipos com ambientes. In: NASS, L.L.; VALOIS, A.C.C.; MELO, I.S. de; VALADARES-INGLIS, M.C. (Ed.). Recursos genéticos e melhoramento - plantas. Rondonópolis: Fundação MT, 2001. p.673-713.

CROSSA, J.; FOX, P.N.; PFEIFFER, W.H.; RAJARAM, S.; GAUCH, H.G. AMMI adjustment for statistical analysis of an international wheat yield trial. Theoretical and Applied Genetics, v.81, p.27-37, 1991.

CRUZ, C.D.; CARNEIRO, P.C.S. Modelos biométricos aplicados ao melhoramento genético. Viçosa: UFV, 2003. 585p.

DAROS, E.; BESPALHOK FILHO, J.C.; ZAMBON, J.L.C.; OLIVEIRA, R.A. de; RUARO, L.; WEBER, H. RB966928 early maturing sugarcane cultivar. Crop Breeding and Applied Biotechnology, v.10, p.278-282, 2010. 
DUARTE, J.B.; VENCOVSKY, R. Interação genótipo $\mathbf{x}$ ambientes: uma introdução à análise AMMI. Ribeirão Preto: Sociedade Brasileira de Genética, 1999. 60p. (Série monográfica, 9).

EMATER. Rio Grande do Sul/ASCAR. Plano anual de trabalho da Emater RS/ASCAR: 2012. 2.ed. rev. atual. Porto Alegre: Emater/RS: Ascar, 2011. 75p. (Série relatórios).

GABRIEL, K.R. The biplot graphic of matrices with application to principal component analysis. Biometrika, v.58, p.453-467, 1971.

GAUCH, H.G.; ZOBEL, R.W. Optimal replication in selection experiments. Crop Science, v.36, p.838-843, 1996.

GUERRA, E.P.; OLIVEIRA, R.A. de; DAROS, E.; ZAMBON, J.L.C.; IDO, O.T.; BESPALHOK FILHO, J.C. Stability and adaptability of early maturing sugarcane clones by AMMI analysis. Crop Breeding and Applied Biotechnology, v.9, p.260-267, 2009.

INSTITUTO BRASILEIRO DE GEOGRAFIA E ESTATÍSTICA. Levantamento sistemático da produção agrícola. Disponível em: $\quad<$ http://www.ibge.gov.br/home/estatistica/indicadores/ agropecuaria/lspa/lspa_200902_4.shtm>. Acesso em: 9 dez. 2010.

LIN, C.S.; BINNS, M.R. A superiority measure of cultivar performance for cultivar $\mathrm{x}$ location data. Canadian Journal of Plant Science, v.68, p.193-198, 1988.

MALUF, J.R.T.; WESTPHALEN, S.L.; MATZENAUER, R.; MALUF, D.E. Zoneamento agroclimático atualizado para a cultura da cana-de-açúcar no Estado do Rio Grande do Sul, visando à produção de açúcar e álcool. Porto Alegre: FEPAGRO, 2008. 78p. (FEPAGRO. Boletim técnico, 18).

MANZATTO, C.V.; ASSAD, E.D.; BACCA, J.F.M.; ZARONI, M.J.; PEREIRA, S.E.M. Zoneamento agroecológico da cana-de-açúcar: expandir a produção, preservar a vida, garantir o futuro. Rio de Janeiro: Embrapa Solos, 2009. 55p. (Embrapa Solos. Documentos, 110).

MARJANOVIĆ-JEROMELA, A.; NAGL, N.; GVOZDANOVIĆ-VARGA, J.; HRISTOV, N.; KONDIĆ-ŠPIKA, A.; VASIĆ, M.; MARINKOVIĆ, R. Genotype by environment interaction for seed yield per plant in rapeseed using AMMI model. Pesquisa Agropecuária Brasileira, v.46, p.174-181, 2011.
MELO, L.C.; MELO, P.G.S.; FARIA, L.C. de; DIAZ, J.L.C.; DEL PELOSO, M.J.; RAVA, C.A.; COSTA, J.G.C. da. Interação com ambientes e estabilidade de genótipos de feijoeiro-comum na Região Centro-Sul do Brasil. Pesquisa Agropecuária Brasileira, v.42, p.715-723, 2007.

OLIVEIRA, A.B. de; DUARTE, J.B.; PINHEIRO, J.B. Emprego da análise AMMI na avaliação da estabilidade produtiva em soja. Pesquisa Agropecuária Brasileira, v.38, p.357-364, 2003.

PACHECO, R.M.; DUARTE, J.B.; VENCOVSKY, R.; PINHEIRO, J.B.; OLIVEIRA, A.B. Use of supplementary genotypes in AMMI analysis. Theoretical and Applied Genetics, v.110, p.812-818, 2005.

PEREIRA, A. da S.; COSTA, D.M. da. Análise de estabilidade de produção de genótipos de batata no Rio Grande do Sul. Pesquisa Agropecuária Brasileira, v.33, p.405-409, 1998.

RAIZER, A.J.; VENCOVSKY, R. Estabilidade fenotípica de novas variedades de cana-de-açúcar para o Estado de São Paulo. Pesquisa Agropecuária Brasileira, v.34, p.2241-2246, 1999.

RAMALHO, M.A.P.; FERREIRA, D.F.; OLIVEIRA, A.C. de. Experimentação em genética e melhoramento de plantas. Lavras: Ufla, 2000. 326p.

SAS INSTITUTE. SAS/STAT: user's Guide. Version 9.2. Cary: SAS Institute, 2009. 7869p.

SILVA, M. de A. Interação genótipo x ambiente e estabilidade fenotípica de cana-de-açúcar em ciclo de cana de ano. Bragantia, v.67, p.109-117, 2008.

SILVA, M. de A.; LANDELL, M.G. de A.; GONÇALVES, P. de S.; MARTINS, A.L.M. Yield components in sugarcane families at four locations in the state of São Paulo, Brazil. Crop Breeding and Applied Biotechnology, v.2, p.97-106, 2002.

SILVEIRA, L.C.I. Adaptabilidade e estabilidade de clones de cana-de-açúcar no Estado de Minas Gerais. 2011. 60p. Dissertação (Mestrado) - Universidade Federal do Paraná, Curitiba.

ZOBEL, R.W.; WRIGHT, M.J.; GAUCH, H.G. Statistical analysis of a yield trial. Agronomy Journal, v.80, p.388-393, 1988.

Recebido em 15 de janeiro de 2012 e aprovado em 23 de março de 2012

Pesq. agropec. bras., Brasília, v.47, n.4, p.561-568, abr. 2012 